Meta

Journal des traducteurs

Translators' Journal

\title{
Conjecture, conjoncture, ouverture : une vue non déterministe de la traduction (Popper, Kuhn et Feyerabend " revisités ")
}

\section{Laurent Lamy}

Volume 58, numéro 1, avril 2013

URI : https://id.erudit.org/iderudit/1023807ar

DOI : https://doi.org/10.7202/1023807ar

Aller au sommaire du numéro

\section{Éditeur(s)}

Les Presses de l’Université de Montréal

\section{ISSN}

0026-0452 (imprimé)

1492-1421 (numérique)

Découvrir la revue

Citer cet article

Lamy, L. (2013). Conjecture, conjoncture, ouverture : une vue non déterministe de la traduction (Popper, Kuhn et Feyerabend « revisités »). Meta, 58(1), 1-24. https://doi.org/10.7202/1023807ar

\section{Résumé de l'article}

Le présent article situe l'expérience de la traduction et la réflexion traductologique dans le cadre des débats épistémologiques initiés avec les travaux de Popper, Kuhn et Feyerabend. Pour ce faire, nous examinons un échantillonnage de propositions relatives à l'épistémè poppérienne et darwinienne mise de l'avant par Andrew Chesterman, l'incommensurabilité entre les programmes de recherche de Berman et de Toury, l'introduction aux études basées sur le corpus par Mona Baker, en référence à la notion de « troisième code » introduite par William Frawley, et, enfin, le caractère évolutif du protocole élaboré par l'équipe du GRETI pour sa retraduction de Faulkner. Nous sommes ainsi parvenus à trois constats : 1) tout traitement des données dans le cadre de la réflexion traductologique repose sur un savoir $a$ posteriori et doit n'admettre qu'une axiomatisation « faible " liée à l'observation de tendances et de régularités n'obéissant pas nécessairement à une causalité ou à une finalité stricte ; 2) toute proposition élaborée dans ce cadre doit conserver un caractère non déterministe ; 3) la traduction comme expérience et la réflexion qui s'en inspire obéissent à un protocole évolutif. Nous concluons en exposant quelques prémisses d'une épistémologie pluraliste, ouverte, en nous inspirant de l'attitude critique défendue par l'épistémologue autrichien Paul Feyerabend.
Tous droits réservés @ Les Presses de l’Université de Montréal, 2014
Ce document est protégé par la loi sur le droit d'auteur. L’utilisation des services d'Érudit (y compris la reproduction) est assujettie à sa politique d'utilisation que vous pouvez consulter en ligne.

https://apropos.erudit.org/fr/usagers/politique-dutilisation/ 


\title{
Conjecture, conjoncture, ouverture: une vue non déterministe de la traduction (Popper, Kuhn et Feyerabend « revisités »)
}

\author{
LAURENT LAMY \\ Université de Montréal, Montréal, Canada \\ laurent.lamy@umontreal.ca
}

\section{RÉSUMÉ}

Le présent article situe l'expérience de la traduction et la réflexion traductologique dans le cadre des débats épistémologiques initiés avec les travaux de Popper, Kuhn et Feyerabend. Pour ce faire, nous examinons un échantillonnage de propositions relatives à l'épistémè poppérienne et darwinienne mise de l'avant par Andrew Chesterman, l'incommensurabilité entre les programmes de recherche de Berman et de Toury, l'introduction aux études basées sur le corpus par Mona Baker, en référence à la notion de «troisième code» introduite par William Frawley, et, enfin, le caractère évolutif du protocole élaboré par l'équipe du GRETI pour sa retraduction de Faulkner. Nous sommes ainsi parvenus à trois constats: 1) tout traitement des données dans le cadre de la réflexion traductologique repose sur un savoir a posteriori et doit n'admettre qu'une axiomatisation «faible » liée à l'observation de tendances et de régularités n'obéissant pas nécessairement à une causalité ou à une finalité stricte; 2 ) toute proposition élaborée dans ce cadre doit conserver un caractère non déterministe; 3) la traduction comme expérience et la réflexion qui s'en inspire obéissent à un protocole évolutif. Nous concluons en exposant quelques prémisses d'une épistémologie pluraliste, ouverte, en nous inspirant de l'attitude critique défendue par l'épistémologue autrichien Paul Feyerabend.

\begin{abstract}
This paper locates the experience of and the reflection upon translation in the framework of foundational issues raised by the works of Popper, Kuhn and Feyerabend. Then we proceed to a thorough examination of a sampling of propositions related to Andrew Chesterman's popperian and darwinian stance, the incommensurability between Berman's and Toury's research programs, the introduction to corpora studies by Mona Baker, advocating the notion of a "third code" put forth by William Frawley, and, last but not the least, the evolutive nature of the protocol performed by the GRETI team in its new north american french translation of Faulkner. So we came to three conclusions: 1) any processing of data in translation studies is based on an a posteriori knowledge and should be implemented with "soft" axiomatics dealing with trends and regularities without any necessary binding to strong causal or final conditions; 2 ) any proposition formulated in this framework should keep a non deterministic profile; 3 ) translation as experience and self-examination is governed by an evolutive protocol. We conclude by putting forth some premisses of a pluralistic, open, scientific endeavor, joining issue with the critical stance advocated by the austrian philosopher of science Paul Feyerabend.
\end{abstract}

\section{MOTS-CLÉS/KEYWORDS}

faillibilisme, incommensurabilité, évolution, ouverture, fécondité

fallibilism, incommenrability, evolution, widening, fruitfulness 
We have here indeed what may very probably be the most complex type of event yet produced in the evolution of the cosmos.

(Richards 1953: 250)

\section{A ghost in the machine?}

Toute hypothèse de recherche doit pouvoir être soumise à l'épreuve des faits pour être confirmée ou infirmée. C'est là sans doute ce qu'on soupçonne être le handicap majeur, s'il en est, de certaines recherches dans le champ des sciences humaines. À tort ou à raison, on demeure dubitatif, à tout le moins perplexe quant à la scientificité des observations qui y sont effectuées et, a fortiori, quant à la valeur objective des propositions qui y sont avancées. Celles-ci, en effet, du point de vue tant de la sélection de leur échantillonnage que des modes de conceptualisation mis à contribution, ne peuvent faire l'économie de la relativité s'attachant à l'exercice de l'interprétation. Il va sans dire que des disciplines comme la sociologie, l'histoire ou la psychanalyse, plus récemment celle de la traductologie, font une large part à l'interprétation. D'où l'imputation d'une carence en matière d'objectivité, faute d'un instrument de mesure réduisant l'interférence escomptée d'une subjectivité nécessairement impliquée dans l'événement qu'il faut décrire et expliquer, facteur qu'on estime alors préjudiciel à l'obtention de résultats probants.

Mais il s'agit d'un préjugé, car rien n'indique que l'on doive répudier l'agent humain et la part de subjectivité qu'il ne peut manquer d'afficher, l'abstraire du champ de l'expérience pour se prévaloir idéalement (illusoirement) d'une donnée fiable (neutre), mais qui demeure toujours d'ordre phénoménal. D’où ce truisme incontournable: il n'est d'expérience que pour un sujet humain.

Il en va ainsi pour deux raisons, parmi bien d'autres. D'abord, il est acquis depuis Kant (1724-1804) qu'en matière de connaissance et quant à son fondement, nous n'avons jamais accès à la «chose en soi » (Ding an sich), ce qu'il désignait alors comme le noumène, mais seulement au phénomène, à ce qui se manifeste (Kant 1781/1975). Les principes de la connaissance chez Kant, les catégories de l'entendement, sont des formes innées de l'intuition. Qu'est-ce à dire? Ceci: nous ne percevons et, par suite, ne connaissons pas les objets tels qu'ils sont, mais bien tels que nous les concevons. La réalité ne nous est accessible qu'à l'état de phénomène que nous appréhendons à la faveur de jugements dont le principe existe a priori dans notre esprit, puisque les concepts qui y sont mobilisés n'appartiennent pas au domaine de l'expérience et ne sont pas contenus dans les données sensibles.

Première conséquence: nous devons renoncer à l'idée que nous sommes des observateurs passifs qui attendons de la nature qu'elle nous prodigue ses «lumières» et qu'elle imprime en nous ses lois.

La seconde raison est de non moindre conséquence: il est désormais acquis dans le cadre passablement fluctuant de la mécanique quantique, qu'on ne peut plus faire l'économie de l'intervention de l'agent humain, de celui qu'on désigne comme l'«observateur local». La situation se complique si l'on considère que la matière possède des propriétés corpusculaires et des propriétés ondulatoires qui se manifestent simultanément, et que l'on peut isoler et mesurer à la faveur d'une expérience où l'observateur forme avec la grandeur observable un système quantique. C'est-à-dire qu'il est lui-même inclus dans le monde qu'il observe. C'est là l'origine du principe 
d'incertitude, plus précisément celui d' «indéterminité» (Unbestimmtheit), formulé par le physicien allemand Werner Heisenberg (1901-1976). La physique quantique, du moins dans la variante qui a fait époque et constitué depuis lors la nouvelle orthodoxie, soit l'«l'interprétation de Copenhague», décèle et suppose dans son modèle opératoire un lien de réciprocité irréductible entre observateur et observable: l'observable est à l'origine de la sensation chez l'observateur, mais, inversement, l'observateur crée la chose observée (Bohr 1958/1991).

Mutatis mutandis, cette réciprocité irréductible entre observateur et observable peut être rapprochée de la notion de «cercle herméneutique», d'abord esquissée par le philosophe Wilhelm Dilthey (1833-1911), alors qu'il posait les jalons d'une épistémè propre aux sciences humaines, et qui sera ensuite relayée par l'analytique existentiale de Martin Heidegger (1889-1976), pour enfin être reconduite dans la philosophie herméneutique élaborée par Hans-Georg Gadamer (1900-2002). Je tente ici une formulation la plus succincte possible: l'individu appréhende sa propre expérience vécue à la lumière d'un monde qui constitue l'horizon préalable de son expérience et, en revanche, ce monde ne s'ouvre à lui qu'à partir de sa compréhension préalable des données qu'il tire de cette expérience et du sens qu'il leur imprime au gré de ses diverses interprétations (Lamy 2007).

Il m'apparaît indéniable que le traducteur engagé dans sa décision de traduction, que l'on doit envisager comme solution d'un problème, évolue toujours à l'intérieur d'un «cercle herméneutique». L'interprétant agit en fonction de ce qui se prête à son interprétation, mais l'interprété est aussi tributaire de l'horizon de sens dans lequel évolue l'interprétant. La subjectivation implicite à l'acte d'interprétation qui intervient dans la phase de déverbalisation où le traducteur s'affranchit de la forme linguistique du texte source n'est pas exclusivement une manifestation de libre arbitre, mais tombe sous la juridiction des possibles avalisés par son apprentissage de la langue hôte, laquelle coïncide le plus souvent avec sa langue maternelle. Ce qu'on désigne par l'expédient des "automatismes de traduction» ressortit à une sédimentation beaucoup plus profonde et à une incubation beaucoup plus complexe des processus d'apprentissage liés à la configuration d'un monde, d'un horizon de sens construit dans la langue et qui se prolonge nécessairement dans le geste traducteur. Aussi ne saurait-on être surpris ou s'étonner outre mesure que la motion initiale, presque congénitale, de ce geste soit une tentative d'infléchir la dissymétrie vivement pressentie dans l'abord de la source étrangère. Pour être franc, quoi de plus naturel que l'ethnocentrisme, l'hypertextualité ou le platonisme (précession du sens au détriment de la forme) mis en cause par Antoine Berman (1985/1999) ou encore, suivant le binôme introduit par Lawrence Venuti (1995), la naturalisation (domesticating) prenant le pas sur l'étrangéisation (foreignizing)?

\section{La «crise» des fondements comme fondement d'un savoir réfléchi}

Dans le second versant du XIX ${ }^{\mathrm{e}}$ siècle, au moment même où s'annonçaient les signes avant-coureurs présageant de la crise des fondements qui allait ébranler tout l'édifice du savoir et culminer avec l'élaboration de la mécanique quantique et de la cosmologie relativiste au tout début du $\mathrm{xx}^{\mathrm{e}}$ siècle, une distinction quasi congénitale à la perception de la science régnait en maître, comme si elle allait de soi. Il s'agit de la discrimination, bien ancrée historiquement, entre les «sciences de la nature», les 
Naturwissenschaften, promues par l'idéalisme allemand et institutionnalisées ensuite dans les universités, instituts et académies allemands, et, par ailleurs, le domaine de la culture, associé aux «sciences de l'esprit», les Geisteswissenshaften. Suivant une acception devenue canonique, qui n'est autre en réalité qu’un préjugé tenace, la prérogative d'une connaissance objective serait réservée au domaine des sciences de la nature, puisque ce genre de connaissance serait basé sur l'observation de faits empiriques, dont l'analyse est conduite à l'aide d'un raisonnement de type hypothéticodéductif.

Mais cette dichotomie, qui est préjudicielle à un examen plus approfondi de la réalité, ne tient plus la route en considération des mutations radicales survenues dans les conceptions de la réalité physique depuis le début $\mathrm{du} \mathrm{xx}^{\mathrm{e}}$ siècle. Je l'ai évoquée brièvement plus haut, une des conséquences de cette conversion du regard porté sur les composantes élémentaires de la réalité physique ainsi que sur le rôle, plus précisément la localisation de son assesseur essentiel, l'agent cognitif humain, est l'introduction par Werner Heisenberg (printemps 1927) du principe d'incertitude, qui répond à l'insolubilité de la dualité onde-particule et selon lequel l'observation induit le comportement du phénomène observé, de sorte que l'instrument de mesure et l'observateur qui interprète ses données sont compris dans le phénomène. On doit alors faire acte de modestie et se résumer à un déterminisme d'ordre probabilitaire: les relations d'incertitude liées à l'impossibilité de connaître à la fois la vitesse et la position d'une particule à cause de la perturbation induite par le dispositif de mesure sont distribuées sur une fonction de probabilité. Cette condition expérimentale s'est révélée être en fin de compte une propriété intrinsèque de l'objet quantique. Autrement dit, la transition du «possible» au «réel» a lieu pendant l'acte d'observer.

Cette condition expérimentale présente, à mon sens, une singulière ressemblance avec la condition qui prévaut dans la situation de traduction. Quoique beaucoup plus complexe mais apparemment plus aisé à maîtriser, à certains égards, que la manipulation des variables assorties aux flux d'énergie de haute intensité, l'espace de résolution du traducteur est aussi marqué du sceau de l'incertitude. La négociation entre la forme et le fond, tout comme l'opportunité de dénouer l'intrication de la signification aux divers contextes d'énonciation, présente un coefficient de difficulté qui répudie toute solution déterministe sous forme d'équivalence stricte, formelle ou dynamique (voir Nida et Taber: 1969). La décision de traduction, à l'instar des ponctions opérées par le dispositif de lecture de l'objet quantique, est le lieu électif, le momentum où s'effectue la transition du «possible» au «réel». Le traducteur est nécessairement intégré à ce dispositif, qu'il perturbe mais où il ne peut s'abstenir d'interférer car il lui incombe précisément de sélectionner et d'actualiser une solution de traduction, de privilégier une forme en se mesurant à l'impératif du sens. Sans compter que l'écart assumé dans la décision de traduction, son amplitude, ne peut être jaugé qu'a posteriori, dans le tissu total du texte et eu égard à son point d'impact dans l'usage qui en sera fait. Aussi, à la trichotomie introduite par Umberto Eco dans Les limites de l'interprétation, qui distingue la visée de l'auteur (intentio auctoris), la dynamique intrinsèque de l'œuvre (intentio operis) et les attentes du lectorat (intentio lectoris) (Eco 1990/1992: 29-32), j'adjoindrais volontiers l'intentio translatoris, qui en est la mandataire tout en jouissant d'une marge de manœuvre qui doit être calibrée dans la décision de traduction. Tous ces facteurs y interviennent à divers degrés suivant l'expectative qui est mise de l'avant. Évidemment, en m'exprimant de la sorte, 
je hausse les enchères et magnifie le niveau de difficulté aux fins de mon analyse, car quantité de spécimens de traduction agréent une grande fluidité dans la translation du texte source vers la langue hôte. Les déterminations (sémantiques, culturelles, contextuelles, pragmatiques, etc.) jouent à plein, mais l'espace de résolution demeure non déterministe, précisément parce que ce sont des «possibles» et non pas en raison d'une présumée labilité imputable à la denrée subjective. Ceci étant, y a-t-il moyen de formuler des hypothèses valides et viables assurant un gain cognitif dans l'investigation de la dynamique traductionnelle? Certes. Voyons un peu à quelles conditions.

En 1934, le philosophe et épistémologue viennois Karl Popper (1902-1994) réussissait à faire publier sa remarquable Logik der Forschung, traduite en français sous le titre de Logique de la découverte scientifique (1973), où il faisait d'abord valoir l'idée qu'aucun critère logique de vérification ne permet jamais de distinguer une théorie scientifique d'une théorie qui ne l'est pas. Suite à quoi il introduisait son fameux critère de réfutabilité, qu'il intégrait à un vaste système évolutif, de type darwinien, établissant la concurrence des hypothèses. Pour lui, en effet, le seul signe, la seule pierre de touche qui nous permette de discerner et d'évaluer la consistance scientifique d'un énoncé est son aptitude à être falsifié, c'est-à-dire réfuté par des tests empiriques négatifs: "le critère de la scientificité d'une théorie réside dans la possibilité de l'invalider, de la réfuter ou de la tester" (Popper 1986: 75). Autrement dit, la science ne procède et ne progresse pas de certitudes absolues en certitudes absolues, mais d'hypothèses de travail en corrections d'erreurs successives. Bref, la science avance en émettant des conjectures qu'elle tente ensuite de réfuter: la théorie vient donc avant l'expérience et ne constitue par conséquent qu'une hypothèse provisoire, une approximation de la vérité qui est appelée à être corroborée dans la seule et unique mesure où elle est susceptible d'être réfutée.

En 1962, Thomas Kuhn (1922-1996) publiait un ouvrage qui allait lui aussi faire époque, Structure of Scientific Revolutions (1962/1972). Il prenait pareillement acte de la crise fatale qui sonnait le glas de l'épistémologie d'obédience positiviste ou logico-empiriste, mais sur des bases tout à fait différentes de celles qui inspiraient le falsificationnisme poppérien. Il introduisait alors sa notion de paradigm shift, de "changement de paradigme», caractérisant ainsi le saut brusque, inopiné et parfois accéléré, qu'entraîne la soudaine désaffection d'une vision du monde formant jusque lors le statu quo, au profit d'une tout autre perspective dont l'entrée en scène, constituant un événement de portée ontologique, engendre littéralement une mutation globale dans la perception même de la réalité. En l'occurrence, une révolution scientifique constitue une sorte de gestalt switch, un changement brusque, discontinu, dans la perception de la réalité et des paramètres spatio-temporels qui lui sont associés.

Or cette transvaluation soudaine des valeurs associées à une Weltanschauung ayant jusque lors préséance, bref à une «vision du monde» correspondant à un paradigme en vigueur, ne va pas sans une réorientation complète du vocabulaire de base assorti à cet horizon de référence. La question de l'incommensurabilité des paradigmes est intimement liée à celle de la communicabilité. En d'autres mots, l'intronisation d'un nouveau paradigme peut nécessiter la création d'une nouvelle constellation de sens et amener à convenir de l'inconsistance du langage en usage dans le cadre du paradigme antérieur. Il va sans dire que les scientifiques appartiennent à des «communautés de langage et de discours» et que cet engagement revêt une portée ontologique. Loin de 
s'abandonner à un relativisme à tous crins, Kuhn constate tout simplement que l'adhésion à une théorie ne saurait s'expliquer totalement et exclusivement en termes de dialogue rationnel ou d'échange d'arguments dont le seul contenu logique importerait, mais elle est aussi affaire de «conversion du regard» et, par conséquent, ne va pas sans un réaménagement lexical majeur, puisque la relocalisation, parfois vertigineuse, si ce n'est la révocation des paramètres jusque lors prévalents, à la suite de l'éclosion de ce nouvel horizon de référence, rend caducs nombre de concepts et de termes en usage dans le cadre de la théorie antérieure.

Sans doute est-il opportun de se demander si l'incommensurabilité frappant le vocabulaire et la conceptualité dans le "passage à vide» que peut entraîner la «conversion du regard» dans le cas de paradigmes historiquement datés, n'est pas déjà une propriété intrinsèque de notre relation au langage, dont la traduction, l'œuvre de "truchement», serait symptomatique de par la diversité des facteurs qui la rendent pratiquement problématique par nature et la variété des topiques de recherche qui en découlent. Si la discontinuité enregistrée dans la rupture du consensus lié à la prévalence d'un paradigme et le passage à une nouvelle configuration du savoir de pointe posent la question de l'incommensurabilité des lexiques qui leur sont respectivement associés, pareille incommensurabilité peut aussi être observée entre des programmes de recherche contemporains les uns des autres et visant sensiblement le même objet d'étude mais qui, paradoxalement, ne semblent pas pour autant en concurrence ni même en contact les uns avec les autres, car n'occupant pas le même terrain en raison de la spécificité de leur contexte théorique et de leur champ d'application. C'est une question qui mérite d'être examinée, car on la rencontre précisément dans le domaine de prédilection de la réflexion traductologique, qui réunit plusieurs tendances épousant des tangentes parfois difficiles à réconcilier. Je crois aussi que pour ce faire il serait opportun de mettre à profit le gain significatif qui nous a été prodigué en matière d'analyse épistémologique par les travaux de Popper et de Kuhn.

\section{Entia non sunt multiplicanda praeter necessitatem: l'incommensurabilité des paradigmes, l'idée d'un "troisième code» et le principe de parcimonie}

L'interdépendance parfois étroite et très sensible entre la modélisation d'une vision du monde et le champ lexical qui lui est corrélé, bref le problème de l'incommensurabilité des paradigmes et de la traduisibilité des concepts est l'idée que Kuhn va reconduire avec le plus de constance et d'insistance suite à la publication de son maître ouvrage. Sa pugnacité à cet égard va lui attirer nombre de critiques. D’aucuns ont cru bon rapprocher ses thèses de celles que Quine a développées dans Word and Object (Quine: 1960) sur l'indétermination radicale de la traduction et l'inscrutabilité de la référence. D’autres encore l'ont associé aux tenants de l'hypothèse SapirWhorf sur le relativisme linguistique, sinon campé dans une variante à peine mitigée du relativisme tous azimuts auquel on ravale, à tort et sans autre forme de procès, les vues très pénétrantes de Paul Feyerabend.

Il n'est pas lieu ici pour moi de dénouer les fils de ce noeud gordien. J'évoquerai plutôt, brièvement, le parti très fécond qu'en a tiré Siobhan Brownlie dans un article où elle traite de l'incommensurabilité des programmes de recherche mis de l'avant respectivement par Antoine Berman et Gideon Toury, leur problématique inter-tra- 
duisibilité (Brownlie 2003 : 93-120). Elle relève d'abord cet élément important, crucial, des analyses de Kuhn, à savoir que l'incommensurabilité entre des schèmes conceptuels inscrits dans des paradigmes radicalement discontinus, tout comme le problème initial de la traduction entre les langues, est due principalement à l'impossibilité de se prévaloir d'un "tiers langage» (un "troisième code») pouvant servir de point d'ancrage et de comparaison pour arbitrer le «différend». En fait, comme l'observe Brownlie, les cadres de recherche et les horizons de référence de nos deux théoriciens sont affectés d'un indice d'incommensurabilité qui défie toute «conversion»: «Toury would never convert to the Berman paradigm and Berman would never have converted to the Tourian paradigm. Nor can we say that one framework is more intellectually powerful and insightful and will supersede the other" (Brownlie 2003: 95).

Pour cette raison nous avons besoin d'une notion plus forte de l'incommensurabilité (a stronger notion of incommensurability). Elle va la quérir chez Jean-François Lyotard. Pour ce dernier, en effet, il n'existe aucun tribunal, ni tiers parti, pour arbitrer le différend. C'est le point de vue, assez radical, qui sert d'amorce à ses brillantes analyses, menées à bâton rompu, dans Le différend (Lyotard:1983), dont la force et la pertinence, bien que Brownlie omette de le souligner, tiennent à sa mobilisation de l'acuité analytique des outils conceptuels développés dans le cadre de la pragmatique des actes de langage. Lyotard en conclut à l'insolubilité de la différence entre divers "genres de discours», leur hétérogénéité foncière qui ouvre sur un abîme qui ne laisse de se creuser si l'on s'en tient au principe de ces discours qui sont très typés et pour ainsi dire jaloux de leur juridiction respective (la loi, la vérité, le pouvoir, d'où l'exercice de la force de persuasion et autres effets de rhétorique relevant de la pragmatique du discours).

Mais le problème ici, eu égard aux programmes respectifs de Berman et de Toury et nonobstant la pertinence de ce renvoi par Brownlie à l'analytique des genres de discours proposée par Lyotard, est qu'il n'y a pas, à proprement parler, de « différend». Le débat n'a jamais eu lieu. Bref, le problème ne s'est jamais posé. Nous sommes en présence de deux univers parallèles. La base de Berman est l'herméneutique moderne, celle notamment de Paul Ricœur et de Hans Robert Jauss, ainsi que la théorie critique élaborée par l'aréopage des Romantiques allemands évoluant autour de Friedrich Schlegel et de l'Athenäum à Iéna, dont Walter Benjamin est l'héritier avoué. Le fonds de commerce de Toury est la théorie systémique développée par Itamar Even-Zohar, la science empirique et la socio-sémiotique. L’approche de Berman est herméneutique et axiologique, celle de Toury, descriptive et systémique. Le risque est grand d'être confronté à une amplitude irréductible, irréconciliable, tant la disparité entre les deux dispositifs narratifs est accusée. Derechef, nous avons en substance deux lignes d'univers parallèles qui poursuivent leur tracé et décrivent une trajectoire qui défie les relations logiques habituelles d'exclusion, d'inclusion et d'intersection. Poser la question de l'incommensurabilité dans ce cas, c'est pratiquement y répondre, car il n'y a pas à proprement parler de point de comparaison.

Il serait facile, je crois, de cantonner les deux parties de ce "différend " appréhendé, pour l'une, dans une faction «continentale», spiritualiste et essentialiste, et pour l'autre, une faction anglo-saxonne (et autrichienne à la source), de tradition rationaliste et plus volontiers systémique. Étonnamment, Brownlie cède à cette tentation. Pour elle, en effet, la position de Berman est trempée d'une vue essentialiste, 
saturée de renvois à la teneur de vérité des œuvres et de jugements de valeur distribués à gauche et à droite, posture qui se recommande d'une vision unifiée de la traduction, dans son concept comme dans son histoire et dans sa pratique (Brownlie 2003: 100101). Pour Toury, en revanche, l'activité du traducteur est une activité sociale gouvernée par des normes la rattachant à un environnement culturel spécifique et qui sont relatives à une société, une culture et une époque données (Brownlie 2003: 102-103). Pour Berman, toujours suivant l'angle de lecture adopté par Brownlie, l'histoire de la traduction, dont la trajectoire épouse celle des œuvres et de leurs retraductions, vise un idéal canonique qui se perpétue et traverse le temps à l'instar de la quête d'une essence atemporelle. Toury n'a visiblement que faire d'une quelconque essence de l'œuvre et de ses traductions successives ou intermittentes, il n'est concerné que par la description des modes de conformation aux normes et des règles de fonctionnement qui ne visent aucune forme de perfectionnement implicite. Selon Brownlie, Berman accorde une importance exclusive, voire démesurée, à la performance individuelle du traducteur, tandis que Toury ne s'attache qu'à l'analyse des contraintes sociales et systémiques.

Je concède que chez Berman les aspects herméneutiques de la traduction individuelle priment alors que Toury, sans pour autant faire totalement l'impasse sur ces facteurs, est nettement moins concerné par la créativité individuelle, les variations idiosyncrasiques d'une interprétation qui idéalement - c'est ma lecture de Berman - viendrait se fondre dans la courbe de croissance où se concentre la trajectoire hyperbolique appelée à pénétrer l'essence d'une œuvre à travers l'histoire de ses traductions. Car c'est là, en fin de compte, le lieu du «différend», s'il en est. Le propos de Berman comporte aussi, dans sa revendication expresse à l'endroit de la teneur de vérité et de l'essence des œuvres et de leurs traductions, une tonalité axiologique qui est à l'évidence absente de la perspective systémique de Toury, qui se prétend axiologiquement neutre et objective. En substance, chez Berman, qui reconduit à bien des égards le nominalisme qualitatif imprégnant la métaphysique du langage élaborée par Walter Benjamin $(1916 / 1991$; 1923/1997), le destin des traductions suit la ligne de faille où se retrace l'épiphanie fragmentée des degrés de croissance de l'œuvre, l'approximation progressive de son essence à travers le travail de traduction, alors que Toury, ne s'intéressant qu'à des effets de structure, s'efforce tout simplement de mettre en lumière les rouages d'un système d'acteurs sociaux et, pour ce faire, favorise la minutie et la densité de la description dans l'exclusive de tout jugement de valeur.

L'incommensurabilité majeure joue donc sur la valeur de vérité accordée au travail de la traduction ainsi que la valeur ontologique ajoutée que lui attribue Berman. La disparité des paradigmes en cause et celle des lexiques qui leur sont électivement associés se traduit à mon sens dans la nature des inférences qu'ils habilitent respectivement, plus précisément dans les types d'hypothèses qui peuvent être avancées de part et d'autre. Pour anticiper sur des développements de la prochaine section et pour fausser compagnie aux analyses de Brownlie, je dirais volontiers que la démarche de Berman est orientée vers des problèmes de définition et de catégorisation, qui s'autorisent essentiellement d'hypothèses interprétatives. Le paradigme systémique de Toury, qui s'inscrit dans le sillage des travaux d'Even-Zohar, est davantage concerné par des problèmes de généralisation, laquelle repose sur des hypothèses descriptives, bref l'observation cumulative de traits distinctifs, de constantes et de régularités 
pouvant éventuellement se prêter à une axiomatisation sous forme de normes et, par suite, à la formulation d'hypothèses explicatives et prédictives.

Sans doute serait-on tenté d'accorder une plus grande crédibilité à un modèle descriptif qui s'en tient à l'observation rigoureuse de traits récurrents, comparativement à l'espèce de saut hyperbolique, voire à l'espèce de gageure à laquelle pourrait être assimilée l'inférence porteuse d'une hypothèse purement interprétative. Il faut être prudent, sinon plus ouvert, et ne pas préjuger des mérites de l'un ou de l'autre et, surtout, comme le notait plus haut Brownlie, nous ne pouvons pas dire que «one framework is more intellectually powerful and insightful and will supersede the other» (Brownlie 2003: 95). Qui plus est, le domaine d'expérience de la traduction est d'abord un champ d'interprétation et cette condition structurante de la décision de traduction se reflète aussi dans la modélisation traductologique, se prétendrait-elle axiologiquement neutre et objective.

Qu'on me permette ici de donner un exemple, plus précisément d'exposer un faisceau de spéculations à l'intérieur d'un cadre théorique qui me semble bien illustrer ce chevauchement, parfois imperceptible tant le glissement est subtil, entre un protocole de recherche se prêtant à des hypothèses descriptives et qui finit par générer des hypothèses de nature interprétative. Depuis quelques années s'est élaboré dans le domaine de la traductologie un vaste programme de recherche dont le mode d'investigation est de mieux en mieux instrumenté et qui s'attache tout particulièrement à la «langue de traduction», à sa spécificité, soit la méthodologie appliquée à l'étude des corpora (corpus-based methodology). Le balayage déjà réalisé d'une masse critique d'échantillons viendrait corroborer l'hypothèse d'un «troisième code», d'une langue spécifique à la traduction qui, de ce fait, constituerait une «forme unique de communication» dont les études sur le corpus permettraient de mettre en lumière et de mieux comprendre les contraintes, les pressions et les motivations qui influencent spécifiquement la dynamique traductionnelle.

C'est, en gros, brièvement esquissé, l'aperçu que nous en donne mais aussi la thèse que défend Mona Baker (1998) en introduction à un numéro thématique de Meta consacré au développement de cette approche jusque lors inédite ou encore à peine exploitée par la recherche en traductologie. Elle précise: «l'idée que la langue de traduction diffère de la langue courante a été, et est toujours, débattue dans les écrits en dehors des études sur le corpus. Cependant, abstraction faite des travaux sur les études de corpus, aucune étude à grande échelle n'a examiné ce phénomène» (Baker 1998: 481). Forte de ce constat, elle nous rappelle que le premier à avoir parlé de la «langue de traduction» comme possédant sa spécificité propre est William Frawley (1984). Ce dernier suggère en effet que «la confrontation du texte source et de la langue cible pendant le processus de traduction crée ce qu'il appelle un "troisième code" (third code). En d'autres mots, le code (ou la langue) qui évolue pendant la traduction, et dans lequel le texte cible est rédigé, serait unique» (Baker 1998: 481). Baker met ensuite en évidence la valeur heuristique de la notion de «troisième code», tout en la distinguant de celle de "jargon de traduction" (translationese) avec ses connotations péjoratives, et énumère les étapes à suivre pour négocier les contraintes qui pèsent sur l'éventualité de parvenir à une clarification décisive de la problématique en cause. Ce survol à caractère programmatique a pour lointain dessein de corroborer une thèse formulée par Baker d'entrée de jeu comme vecteur ou fil conducteur de la recherche et qui en constitue en quelque sorte le postulat, à savoir que 
[la] traduction est une forme unique de communication linguistique et culturelle parce qu'elle implique bien plus que la simple compréhension des subtilités d'une langue et de la structuration de la langue source et de la langue cible. Les caractéristiques propres à la traduction la rendent donc apte à devenir l'objet d'une discipline indépendante que nous appelons traductologie (Baker 1998: 480).

L'idée de comparer non pas le texte source et la langue cible, mais la facture du texte cible avec celle de textes comparables écrits directement dans la langue cible par des locuteurs indigènes, si je puis ainsi m'exprimer, déplace la réflexion sur la langue de traduction hors des ornières de l'équivalence et du binôme fidélité/liberté, s'engageant alors dans une tout autre optique qui est susceptible de baliser un terrain propre à l'activité du traduire, un terrain de prédilection doté de ses propres paramètres. Il n'est pas difficile de concevoir ici que cette ligne de recherche nourrit, outre le balisage d'un champ d'application concret à la faveur d'une clef heuristique, celle de «troisième code", une visée fondationnelle qui concerne le champ entier de la traductologie.

En effet, en nous relayant la thèse de Frawley (1984) et en y trouvant un appui pour l'idée que la traduction est une "forme unique de communication", Baker (1998) nous monnaye deux thèses assez fortes qui nous font quitter le terrain de la description pour le domaine de l'interprétation. L'hypothèse avancée par Frawley (1984) est valide, suivant le critère de démarcation de Popper (1986: 75), car elle est réfutable et, je le crois, loin d'être corroborée. Celle de Baker (1998), qui a aussi une valeur heuristique, car elle pose directement la question du statut de la traduction, l'est aussi, mais elle est vraiment loin d'être avérée. En fait, ces hypothèses demeurent toutes deux problématiques parce qu'elles débordent largement le cadre de la description, qui est précisément celui des études basées sur le corpus. En revanche, j'estime que ces hypothèses sont valides, car ce sont des conjectures qui ont le mérite d'être ouvertes et nous entraînent sur le terrain fécond d'un questionnement ontologique sur le statut de la traduction. Elles nous permettent aussi de constater qu'il est difficile de s'en tenir au plan strict de la description sans déjà emprunter des voies de traverse pour passer au stade de l'explication qui souvent demeure une forme d'interprétation. Il ne s'agit pas pour autant d'un défaut, d'une défaillance de la théorie, mais une propriété intrinsèque de son fonctionnement.

Pour revenir brièvement à Kuhn (1962/1972), une des thèses qu'il partage avec Popper $(1934 / 1973 ; 1963 / 1986)$ et Feyerabend $(1975 / 1979)$, auquel je viendrai plus loin, est ce qu'il désigne comme la theory-ladenness de toute forme d'investigation, aussi empirique soit-elle. En l'occurrence, il n'existe rien de tel qu'un pur «langage d'observation", une pure description, car toute prospection de données empiriques est «armée» d'une théorie préalable qui «informe» le faisceau d'hypothèses qu'on entend y vérifier à la faveur d'une expérimentation dont les paramètres sont déjà calibrés en fonction de cette théorie. Aussi est-il tout naturel et légitime que Frawley (1984) et Baker (1998) puissent se livrer à une interprétation qui, bon gré mal gré, se veut candidate au statut d'explication en bonne et due forme. Ce saut est souvent accompli à notre insu, de façon presque imperceptible, dans l'agencement des propositions au gré desquelles nous tentons de conforter une hypothèse de départ et dans la poussée des stratégies rhétoriques mises à contribution sous la réserve implicite, mais néanmoins difficile à observer, de ne pas céder à la pétition de principe.

Antoine Berman a lui-même défendu l'idée d'une «troisième langue» dans La traduction et la lettre ou l'auberge du lointain (Berman 1985/1999: 112), évoquant le 
recours au latin par Chateaubriand dans sa traduction du Paradise Lost de Milton. Bien que je ne puisse élaborer ici davantage cette question, plutôt que de caresser l'idée d'une «troisième langue», j'abonderais plus volontiers dans le sens d'une triangulation voire d'une prospection matricielle sollicitant divers facteurs, phonémique, syntaxique, sémantique et tropologique, à l'interface entre la source de la traduction et la solution anticipée dans la langue hôte, dont le point d'impact est pour ainsi dire triangulé avec le point de fuite épousant sur un mode tangentiel ce que Walter Benjamin (1916/1991) désigne comme le devenir ou la «croissance» (Wachstum) des langues. Je souscris d'emblée à la thèse formulée par ce dernier, dès 1916, dans son brillant essai Sur le langage en général et sur le langage des humains: "La traduction est le transfert d'une langue dans l'autre à travers un continuum de métamorphoses. La traduction traverse des continuums de métamorphoses, non pas des zones abstraites d'équivalence et de ressemblance» (Benjamin 1916/1991: 151).

Nous demeurons toujours au sein du même élément, un continuum de métamorphoses qui néanmoins obéit à une dynamique non linéaire, densément stratifiée. Lorsque la traduction s'engage face à une œuvre étrangère elle est confrontée à un organisme complexe qui est la résultante d'un très long processus de maturation. L'œuvre, dans sa facture originelle, forme provisoirement un tout achevé, une espèce d'« entéléchie» au sens aristotélicien, bref une forme en puissance qui est mise en acte au stade critique de son achèvement. Mais sa "facture» demeure ouverte, dans le sens de l'opera aperta suggérée dans un fameux ouvrage de ce nom d'Umberto Eco (1962/1965): sa finalisation ou sa complétude, qui est aussi la marque de sa finitude, n'est pas que signe d'étanchéité mais de perméabilité, d'ouverture la rendant susceptible d'encourir diverses transformations qui, par exemple, tel que l'a proposé Kitty van Leuven-Zwart, notamment dans son modèle comparatif, peuvent être de l'ordre de la modulation, ou de la modification ou de la mutation (van Leuven-Zwart 1989: 159-169).

Il n'est guère besoin, à ce titre, de s'en remettre à un "troisième code», sinon de se prévaloir des offices d'un métalangage. L'évolution des langues, qui est tributaire des percolations, des fractures et des synergies opérées dans la gestation des œuvres où converge leur point de fuite, forme un continuum, une espèce de palimpseste dont les couches superposées ont été investies et trempées du labeur multiséculaire de la traduction, laquelle agit comme vecteur de métamorphoses. La difficulté apparente rencontrée dans la solution d'un problème de traduction n'est pas une limite qui rançonne l'universalité de la forme encourant un déficit dans le transfert d'un contenu ou d'une teneur de sens liés à la substance d'une expression. À mon sens, cette difficulté est un problème de perception, et non pas d'essence: les traductions n'ont pas à être ontologiquement différentes de l'original, mais le fait est qu'elles sont souvent perçues comme telles.

Personnellement, je situerais le problème majeur auquel est confrontée la réflexion traductologique au niveau du critère d'engagement ontologique, plus précisément le degré ou la force des inférences que nous pouvons produire au sujet des multiples aspects impliqués dans une décision de traduction. Cette problématique concerne entre autres le partage des universaux de forme et des universaux de substance. Par exemple, il nous est loisible d'y aller d'inférences beaucoup plus fortes et bien maîtrisées pour ce qui est de la forme des langues, alors que leur substance admet tout un spectre de variables très typées et un ensemble de structures marquées dont 
l'amplitude et la sensibilité au contexte culturel et social sont nettement plus difficiles à investiguer et à gérer à l'aide d'hypothèses fortes.

J'estime pour ma part, dans la foulée d'une observation judicieuse de Noam Chomsky, qu'en matière de traduction il y a une incommensurabilité incorrigible quant au partage des universaux de substance, alors que les universaux de forme jouissent d'une prégnance diffuse dans la majorité des langues connues (Chomsky 1965/1971 : 49). Les universaux de forme, qui concernent la charpente commune à presque (mais pas) toutes les langues, ne semblent guère poser problème car on peut aisément en isoler le noyau, le repérer et le voir à l'œuvre dans quantité de langues (mais jamais dans toutes les langues). En revanche, la substance même de la langue, qui imprègne tous les niveaux de l'expression, ne semble guère donner prise qu'à des intuitions sporadiques, qui sont loin de pouvoir constituer, du moins de façon appréciable, un modèle ou une matrice de générativité viable pour la traduction. La raison en est presque triviale mais oh combien diffuse: la maîtrise d'œuvre de la traduction n'a de cesse d'être confrontée à la composante pragmatique et fortement individuée des rouages particuliers à chaque langue, aussi bien la performativité des actes illocutoires et perlocutoires véhiculant certaines attitudes propositionnelles que la prédilection de nature idiomatique pour certains tropes ou tournures métaphoriques.

$\mathrm{Si}$, comme Kant l'a soutenu, toute connaissance est modelée par notre esprit (Kant 1781/1975), en revanche, cette disposition, cette aptitude cognitive qui est nôtre, ne produit pas des certitudes, mais seulement des conjectures. Qui plus est, celles-ci ne seront jamais définitivement prouvées, car leur propriété intrinsèque consiste à être constamment susceptibles de se voir réfutées à la faveur d'une expérience contradictoire. Nos certitudes, en fin de compte, ne portent que sur ce qui est faux. La science, dans son essence même, est une entreprise de doute systématique alliant la hardiesse des hypothèses à la conception de dispositifs expérimentaux les plus minutieux et à l'adoption de stratégies opératoires visant à l'épuration de l'appareil de preuves, ce que j'ai désigné plus haut, dans l'intitulé de cette section, comme le principe de parcimonie. La formulation la plus connue et la plus succincte de ce principe, que l'on désigne aussi comme le "rasoir d'Ockham», est attribuée à un moine franciscain et docte médiéval, Guillaume d'Ockham (XIV siècle), bien que l'on n'en trouve nulle trace dans ses écrits: «Entia non sunt multiplicanda praeter necessitatem», littéralement: «Les entités ne doivent pas être multipliées par delà ce qui est nécessaire».

\section{Relativité de toute ontologie: le caractère évolutif de la recherche}

Dans un ouvrage d'une grande fécondité heuristique, Memes of Translation, le traductologue Andrew Chesterman (1997) mobilise avec beaucoup de tact et de circonspection le modèle poppérien, selon lequel toute théorie se présente comme une tentative de résolution de problème, soumise ensuite à un processus d'élimination d'erreur qui, une fois enregistrée et corroborée, à un degré élevé ou moindre suivant le critère épistémologique en cause, va ouvrir la donne pour la formulation d'un nouveau problème. Cette tangente évolutive de l'épistémologie poppérienne est ensuite jumelée à l'idée développée par l'éthologue britannique Richard Dawkins (1976) au onzième chapitre de son fameux ouvrage The Selfish Gene, idée selon laquelle la culture, à l'instar des gènes en biologie, génère des entités «mimétiques » appelées memes par Dawkins et qui se reproduisent, se diffusent et tendent à s'incrus- 
ter et à se perpétuer dans le champ culturel jusqu'à ce qu'elles soient supplantées par d'autres et ainsi vouées à l'obsolescence, sinon à une mutation marquée. Il en va des idées comme des gènes: il en est qui sont voués à l'extinction ou à une bien brève prestation, et d'autres, qui se veulent des replicators plus tenaces, donc qui se répandent et prolifèrent par voie de duplication et d'adaptation évolutive.

L'idée de mème de la traduction, que je traduis par «constante mimétique», est d'abord et avant tout un outil conceptuel. Chesterman (1997) le définit comme une « unité de transmission culturelle» en compétition avec d'autres unités en circulation, qui peuvent être plus anciennes ou plus profondément ancrées, sinon tout nouvellement apparues et tentant de faire pièce à celles qui sont régnantes, éventuellement de les déloger. Les idées se répandent, se diffusent et se reproduisent comme les gènes. La stratégie de Chesterman, de son propre aveu, est de proposer une solution de rechange, une alternative à la métaphore du transfert qui sert à définir traditionnellement l'acte de traduction. À l'idée de diffusion est corrélée celle de mutation: les traducteurs ne sont pas des agents de conservation destinés à préserver le sens, mais des agents de changement qui propagent en transformant. Les normes ne peuvent alors être abordées que sur un mode descriptif et non prescriptif, puisqu'elles sont les excroissances d'un consensus en constante évolution qui redistribue les paramètres à l'aune desquels on juge de la qualité d'une traduction. Il y a rupture et chevauchement de ces stades évolutifs dont le capital conceptuel s'accumule et prolifère tout en se délestant d'éléments ou de perspectives qui perdent de leur pertinence dans la mouvance des nouvelles directions imprimées avec l'enchâssement de «mèmes» novateurs.

Dans Vers une traductologie poppérienne, Chesterman (2006) fournit et explicite la base théorique de ses options. Il préconise une pratique de la traductologie basée sur la formulation et la validation d'hypothèses dans la ligne de pensée de Popper. À partir d'un problème initial, on formule une théorie provisoire sous forme d'hypothèse qui sera ensuite testée, vérifiée, au besoin ajustée sinon réfutée. Ce processus d'élimination des erreurs conduit à la formulation d'un nouveau problème, face auquel toute nouvelle hypothèse demeure toujours une conjecture agréant divers degrés de plausibilité. Comme le précise Chesterman, cette façon de procéder ne relève ni de l'induction ni de la déduction:

On ne commence pas avec des faits ou des données, ni avec une idée théorique, mais avec un problème, une question. Cette méthode se base sur ce que Charles Peirce appelle l'abduction: c'est-à-dire le processus logique par lequel on suggère, on suppose, on envisage des possibilités, et ensuite on soumet ces possibilités à l'examen, à l'épreuve. (Chesterman 2006: 2)

Il y a divers types d'hypothèses, qui sont avancées en fonction d'une question au départ de la recherche. Chesterman (2006) en retient quatre: des hypothèses interprétatives pour les questions de définition et de catégorisation; des hypothèses descriptives pour des questions de généralisation ou la quête d'universaux de la traduction; des hypothèses explicatives, qui traitent des relations de causalité entre textes et contextes, en rapport à la culture et à la cognition; des hypothèses prédictives, qui examinent les conditions conduisant à une traduction acceptable ou non acceptable, recevable ou irrecevable dans la culture cible, son impact à court ou à long terme, etc. Bien que Chesterman ne sente pas le besoin de le spécifier, il va de soi que cette nomenclature, très schématique, peut se prêter à divers chevauchements, 
l'important ici étant de ne pas se méprendre sur la nature d'une hypothèse, de pouvoir mesurer sa pertinence en regard du problème soulevé et, surtout, de garder le sens des proportions quant à l'étendue de sa validité, bref son enjeu et sa portée.

Les hypothèses interprétatives sont les plus fréquentes et partout présentes, car elles soutiennent toute analyse conceptuelle, tout essai de définition ou de catégorisation, qui constitue le préalable à tout exercice de réflexion. Les hypothèses descriptives visent à la généralisation, qu'on pourrait assimiler à une forme d'induction. Elles tentent d'isoler et d'abstraire un trait commun observable dans une masse critique d'échantillons de traduction. Mais là encore, elles devraient s'attacher exclusivement à l'observation de tendances et de régularités pouvant figurer à titre d'universaux de la traduction, sans pour autant en faire une loi ou une norme de causalité stricte, qui est davantage l'apanage des hypothèses explicatives et prédictives. Ces dernières reposent effectivement sur l'examen des conditions causales favorisant l'obtention de tel ou tel autre résultat. Derechef, même avec ce genre d'hypothèses, il faut, du moins pour ce qui est de l'objet visé par la réflexion traductologique, conserver un espace de résolution ouvert à une problématisation ultérieure et garder à l'esprit qu'il s'agit toujours de conjectures susceptibles d'être validées, réfutées ou corroborées, et admettre divers degrés de plausibilité.

Le choix des variables à retenir pour valider une hypothèse est non moins crucial. Comme le stipule Chesterman, «une hypothèse propose un lien entre deux entités, c'est une proposition relationnelle» (Chesterman 2006: 6). Une hypothèse interprétative repose sur un lien conceptuel où une notion renvoie à une autre, s'interprète à travers elle. Dans le cas des hypothèses descriptives aussi bien qu'explicatives ou prédictives, ce lien est empirique et repose sur des variables sélectionnées comme traits distinctifs du texte source ou du texte cible, qui concernent le profil de ce lien, ou qui sont sinon à l'extérieur de ce lien, dans le contexte où il est élaboré. Nous avons donc des variables de profil, qui peuvent s'attacher par exemple à la forme linguistique d'une traduction (la fréquence des propositions relatives, la densité lexicale ou l'usage de termes dialectaux, etc.), et des variables de contexte, qui couvrent un très large spectre, en amont, dans les conditions causales, ou en aval, dans les effets anticipés, de la décision de traduction, qui sont relatifs à son environnement spatial, temporel, culturel ou cognitif. Les variables de profil sont nettement plus stables que les variables de contexte, encore qu'il faille se garder de faire peser sur ces dernières l'hypothèque ou le soupçon d'un relativisme si élastique qu'il finit par s'étioler dans une balkanisation d'univers de sens confinés à leur aire culturelle, comme c'est le cas avec la variante «forte» de l'hypothèse Sapir-Whorf.

Si la sélection des variables peut se révéler un facteur sensible dans une stratégie de recherche, on constate aussi qu'il peut y avoir confusion sur la nature des hypothèses. On peut affirmer ceci en croyant affirmer cela, ou méjuger de la force de l'argumentation mise à contribution, ou y aller d'une inférence alors que l'état de la preuve n'agrée qu'une supposition, ou inversement, monnayer une supposition où se dissimule une inférence désireuse d'emporter la conviction. Ce n'est qu'un échantillon restreint des possibilités de confusion sur l'enjeu et la portée des hypothèses. Cette question touche au critère d'engagement ontologique, qui détermine le degré ou la force d'une inférence et la consistance d'une argumentation, son verrouillage ou sa perméabilité à des alternatives, une problématique sur laquelle je ne peux m’étendre plus longuement ici. 
Cette série d'observations, la présentation des thèses de Popper (1934/1973; 1963/1986) et de Kuhn (1962/1972) aussi bien que les examens successifs de divers patterns d'interprétation et modèles d'analyse, m'ont amené à formuler ce qui, faute de constituer des hypothèses formelles, peut s'assimiler à des constats qui sont au nombre de trois:

1. Tout traitement des données dans le cadre de la réflexion traductologique repose sur un savoir a posteriori et doit n'admettre qu'une axiomatisation «faible» liée à l'observation de tendances et de régularités sur lesquelles pèse un certain nombre de contraintes mais qui ne se prêtent pas nécessairement à la formulation de normes causales au sens strict ou à la supervision d'une téléologie implicite;

2. Toute proposition élaborée dans ce cadre et avec ce type d'objet, en l'occurrence l'agency du traducteur, le processus dans lequel il est engagé et le produit qu'il est habilité à livrer sous telles ou telles autres conditions causales et en vue de telle ou telle autre finalité, doit conserver un caractère non déterministe;

3. La traduction comme expérience et la réflexion qui s'en inspire obéissent à un protocole évolutif: c'est un trait auto-réflexif qui se situe dans la ligne du faillibilisme défendu par Popper et qui implique, par exemple, que les propositions que j'avance ici peuvent et doivent être corroborées ou réfutées, amendées ou ajustées, car elles n'ont rien de définitif et une bonne part de leur valeur vient de leur incomplétude et du supplément d'interprétation qu'elles sollicitent.

Ce schème est évidemment ouvert. Ces propositions découlent en effet de ce que je considère être un postulat essentiel, à savoir la relativité de toute ontologie et, par voie de conséquence, le caractère évolutif de toute recherche. Je devrais sans doute m'en expliquer un peu.

Par axiomatisation «faible», j'entends un régime de règles qui, tout en se reportant à des variables de profil, va se révéler beaucoup plus souple et nettement plus sensible aux variables de contexte. L'indice de relativité est aussi plus accentué, non pas en raison d'une labilité des structures, mais, si je puis dire, faute d'une qualification plus précise, de la mobilité des paramètres. Pareille relativité n'est pas qu'une propriété adventice liée à l'insuffisance ou à l'imprécision des modes d'investigation ou des méthodes d'analyse, mais est ressentie à la source, dans la "génétique» des langues comme «formes de vie» dont la fonction est de signifier, et le moteur de cette signification, l'usage, comme l'a démontré Wittgenstein (1960: 311, §43). L'usage consolide l'adoption d'une forme qui, en principe, ne procède d'aucune nécessité stricte, causalité ou finalité autre que sa valeur d'usage. L'habitus consolidé par l'usage est pour ainsi dire déverrouillé et exhibé, mis à découvert dans l'espace de la traduction qui agit comme un "révélateur», une matrice ouverte qui désubstantialise la forme et la renvoie à son inscrutabilité foncière, à son pur arbitraire.

Qu'est-ce à dire? Ceci: dans la traduction, la forme consolidée par l'usage, par l'habitus qui lui confère son extension, sa capacité de référer à un signifié partagé par une communauté de locuteurs, est confrontée à son propre arbitraire en regard d'une forme étrangère qui relève aussi d'un usage électif conforté par un habitus et ayant force de loi dans son contexte natif, mais qui ne peut davantage être porté à l'actif d'une causalité ou d'une finalité stricte. Je crois que le partage d'une quantité appréciable d'expériences communes à tous les locuteurs de toutes les langues en usage fournit une assiette ontologique suffisante pour les fins auxquelles s'emploie un traducteur, mais, en revanche, la diversité des cas de figure susceptibles de transiter dans 
l'interface traductionnelle est inépuisable et répudie toute axiomatisation «forte» des stratégies de traduction.

En effet, l'économie symbolique qui est à la base de la formation d'une langue ne devient «motivée» qu'au gré d'une longue incubation de l'arbitraire du signe dont l'on ne peut remonter le fil, nonobstant les spéculations sur les archétypes psychiques ou universaux de l'inconscient, ou encore la présumée proximité onomatopoiétique du signe et du son, sinon sa composante iconique, mimétique ou idéogrammatique d'origine. Le degré de complexité encodé dans l'usage d'une langue à point donné de son évolution oppose une résistance qui met au défi toute schématisation univoque de son fonctionnement.

L'idée d'une axiomatisation "faible», me dois-je de le préciser, ne signifie en aucune façon qu'elle soit dépréciée ou minorisée en regard d'une axiomatisation «forte». Tout ce qu'elle indique, c'est que le «domaine de réalité » qu'elle vise est beaucoup plus difficile à appréhender, à décrire et à qualifier que celui qui se prête à une axiomatisation forte. Par exemple, l'objet visé par le questionnement éthique est incomparablement plus difficile à axiomatiser que les lois qui gouvernent le comportement des phénomènes susceptibles de faire l'objet d'une investigation dans le domaine des sciences physiques. Nous pouvons nous entendre sur ce qui est mal, mais il est beaucoup plus difficile de statuer et d'obtenir un consensus sur ce qui est bien.

J'en conviens: l'expression «protocole évolutif» est une excellente candidate au statut d'oxymoron. C'est tout à fait compréhensible. Par principe ou par définition, la notion de "protocole» réfère à quelque chose de contraignant, de valeur statutaire, passablement rigide et parfois inflexible, quelque chose justement qui serait peu susceptible d'évoluer. C'est l'idée d'«agir dans les formes». Ici évidemment, je réfère à un protocole de recherche et non aux règles d'une "étiquette» mondaine. Or les «formes» de la recherche, en science physique ou humaine, depuis le début du siècle dernier, ont dû s'adapter à des objets d'étude qui affichent des propriétés les dotant d'un haut indice d'instabilité. Le ressac s'est progressivement fait sentir dans la conception des protocoles de recherche, la modélisation des théories, la gestion des hypothèses et l'administration de la preuve qui ont dû se modeler sur leur objet et acquérir un profil plus dynamique, de caractère évolutif. Bien que je croie aux vertus de la théorie, j'estime qu'il n'est meilleur mode d'exposition que son application.

Un chantier exemplaire qui met en ouvre cette tangente évolutive dans la prospection, la révision et la reformulation des hypothèses de travail est l'ouvrage dirigé par Annick Chapdelaine et Gillian Lane-Mercier (2001), Faulkner. Une expérience de retraduction. On nous y présente un post-mortem très étoffé et fort bien argumenté de l'expérience de retraduction de deux chapitres du roman The Hamlet de Faulkner menée par le Groupe de recherche en traductologie (GRETI) de l'Université McGill. Nous accédons au laboratoire réflexif d'un itinéraire de recherche qui, doté d'un protocole de départ ouvert, a dû bifurquer, réviser ses stratégies, se transformer et ainsi amender ses visées au fil de réglages et de réajustements ponctuels certes, mais parfois aussi près d'un seuil critique quant à la gestion globale des décisions de traduction.

Je résume d'entrée de jeu: il s'agit de recréer l'organicité du texte faulknérien, bref traduire la spécificité, la signifiance, l'«étranger» du texte de départ à la faveur d'un exigeant et délicat travail sur la «lettre», tel que préconisé par Antoine Berman 
(1985/1999), à qui d'ailleurs est dédié ce travail. Certaines décisions liminaires, loin d'être simplement cosmétiques, portaient aussi bien sur la gestion des passages à niveau entre narration et segments dialogués, afin de garder intacte la disposition du texte de départ et de conserver l'emboîtement des voix, que, par ailleurs, le parti pris de ne pas franciser, en accord avec leur visée non annexionniste, certains éléments (toponymiques, etc.) de l'univers faulknérien, qui en fin de compte ont acquis une valeur emblématique suffisamment éloquente pour qu'on se dispense de les traduire. Guère difficile de deviner qu'il s'agissait entre autres, bien légitimement je crois, de ré-américaniser le texte de Faulkner dans une livrée d'expression française dont la texture allait requérir une sensibilité accrue aux ressources idiomatiques d'un vernaculaire enrichi de sa migration vers le Nouveau Monde et qui offre en sus, comme plus-value souvent négligée, la thésaurisation d'archaïsmes savoureux, souvent dotés d'une singulière puissance d'évocation, hérités de l'époque médiévale et conservés dans ces véritables niches d'écologie linguistique que sont les terroirs.

Forte de ces prémisses, l'équipe du GRETI a commencé à développer son protocole de traduction. La charge sociolectale concentrée dans les dialogues a été ciblée de façon plus soutenue. Et pour en restituer la teneur le recours au vernaculaire s'est très tôt imposé de lui-même. Mais cette décision initiale a donné lieu à une phase jubilatoire de courte durée, marquée par un excès de vernacularisation, qui s'est néanmoins révélée cruciale, car elle a provoqué une réflexion sur la lisibilité recherchée ainsi que le destinataire idéal. Une contrainte liée à la lisibilité escomptée ne pouvait être éludée: la tradition normative et institutionnelle de l'usage du français écrit est notoirement plus rigide et codifiée que celle qui prévaut pour la langue écrite anglaise. En gros, l'équipe du GRETI a progressivement délaissé, tout en suivant les fluctuations sociolectales de près, les indices phonétiques et morphologiques, en faveur d'une dominante lexicale. Leur progression, qui est fort bien illustrée par un échantillonnage des diverses révisions encourues, a connu l'évolution suivante: 1) survernacularisation phonétique et morphologique; 2) début de dévernacularisation: compensation par le lexical; 3) recours nettement accru au lexical.

Ces réajustements ont fourni l'occasion d'explorer les phénomènes sociolectaux microtextuels dans la perspective de leur pertinence. Ensuite, d'observer chez les personnages dotés d'un idiolecte nettement différencié des fluctuations expressives qui sont tributaires de la situation d'énonciation. En outre, les stratégies discursives - modulations de la voix, stratégies rhétoriques, techniques narratives - dépassent le contexte immédiat de l'univers diégétique, le récit proprement dit, et entretiennent des rapports étroits avec la narration extradiégétique, en l'occurrence la perspective du narrateur omniscient.

Mais, comme le dit l'adage, «le diable est dans les détails». Détails qui nous révèlent parfois une sédimentation beaucoup plus dense de la trame sous-jacente à l'œuvre, tissée de renvois obliques qui agissent comme de lointains sémaphores, des clignotants faibles qui une fois entr'aperçus nous découvrent une tout autre cartographie des instances narratologiques et dialogiques. Cet écheveau de difficultés aura donc enclenché de la part de l'équipe du GRETI une effervescence traductionnelle qui s'est soldée, lors d'un premier jet, par un abus systémique de tentative de fusion à l'original, calquant la scansion de la phrase faulknérienne et générant assez vite une série d'automatismes verbaux qui, en seconde lecture, se sont révélés stériles. En fait, la confusion entre le «marqué» et le «nécessaire» a donné prise à une systématicité 
abusive qui brouillait l'indice de lisibilité escompté de la part d'une traduction qui brille autant par sa rigueur que par sa fluidité dans la langue hôte. La tentation de la symbiose ou de la fusion utérine avec les ressorts idiomatiques et la prosodie mis en œuvre dans la poétique faulknérienne devait aussi être conjurée. Ainsi, le processus de relecture de cette première mouture, sans l'original en regard, a permis de recalibrer l'orientation des stratégies de traduction et de restituer son autonomie au geste traducteur, car le premier jet, exaltant la littéralité, ne parvenait pas à faire texte. Une matière première aussi sédimentée, aux veines enchevêtrées, commandait donc un constant retour au banc d'essai des hypothèses de départ. C'est pourquoi, comme le l'ai suggéré, on peut parler d'un "protocole évolutif».

Impossible de s'attaquer à un tel projet sans disposer d'une théorie préalable, d'une grille de lecture jaugeant et anticipant ses conditions de réussite. Impossible de ne pas y aller aussi d'un pari de nature herméneutique. Mais ce n'est pas suffisant. C'est là qu'intervient la série de tests négatifs, les interrogations, les refontes, les relances et, parfois, la répudiation d'une stratégie de traduction qu'on estimait porteuse. Ces oscillations, phases de latence et d'incubation, d'exaltation et précipitation momentanées, qui consistent à projeter des hypothèses, à les tester, à les abandonner ou à les affiner, donnant parfois jusqu'à l'impression d'opérer sur un terrain miné, sont typiques d'une pratique éclairée de la science.

Je m'explique. Un projet de traduction ne fonctionne pas autrement, par exemple, qu'un dispositif de lecture d'un flux d'énergie de haute intensité dans un laboratoire de physique. Derechef, au risque de détonner, je dresserais volontiers un parallèle avec une formule dont j'ai usé plus haut dans ma discussion du principe d'incertitude de Heisenberg, où l'observation induit le comportement du phénomène observé, en sorte que la transition du «possible» au «réel» a lieu pendant l'acte d'observer. J'estime que la décision de traduction, ou le choix d'une solution de traduction, loge à la même enseigne: l'un des possibles du texte source se révèle et se «réalise», devient réel dans la mise en phase qu'implique l'acte de traduction qui, à son tour, met simultanément à découvert tant le passif que l'actif de la langue traduisante. Il n'en va pas autrement dans cette "épreuve de l'étranger» qu'est la traduction: elle offre une lucarne privilégiée pour appréhender l'être-langue de la langue, à savoir que la langue qui m'est propre, «maternelle» ou autre, celle dans laquelle il m'est loisible de traduire, la langue hôte, ne m'apparaît réellement que dans l'espace traductionnel, interface transfrontalière où le "monde possible» configurable par une langue lui révèle ses propres limites et met à l'épreuve sa plasticité conceptuelle et poétique.

Dans le projet de retraduction de Faulkner, l'épreuve décisive est la modulation traductionnelle d'une oralité opérant sur plusieurs registres emboîtés les uns dans les autres, qui se parodient mutuellement, configurant une microcosme qui est un univers en soi. C'est ce que démontre à l'envi la très belle contribution d'Annick Chapdelaine intitulée "Traduire l'incantation de l'œuvre: Le Hamlet de William Faulkner» (Chapdelaine 2001: 83-129). Je ne peux m'y attarder que brièvement, ne serait-ce que pour faire écho à la fine acuité de ses analyses.

Chapdelaine (2001) inscrit d'emblée l'entreprise de son groupe de recherche dans la foulée des directives à saveur axiomatique, mais d'une grande souplesse, formulées par Berman $(1995$; 1985/1999) au fil de sa réflexion sur l'expérience de la traduction, où prime notamment ce qu'il désigne comme le «travail sur la lettre». Loin de se cloisonner dans la plate littéralité et de verrouiller tout élan créatif de la part du 
traducteur, ce travail consiste plutôt, dans le cas qui nous occupe, à transmuer les traits langagiers et poétiques fondamentaux de l'écriture faulknérienne dans la constellation langagière qui est nôtre, l'univers francophone et franco-québécois. La clef de cette ouverture sur le texte est l'oralité qui insuffle l'écriture faulknérienne et qui lui confère sa "portance». Viser l'organicité même du texte faulknérien et la redistribuer dans une facture où entre en résonance la vibrante oralité de sa prosodie n'est pas la simple reproduction de la distribution factuelle des mots et des phrases de l'original, mais la traduction du système global de ses idiotismes et de ses structures narratives et dialogiques.

L'organicité du texte faulknérien, la texture même de sa textualité, aura tôt fait de mettre en évidence l'importance de la polyphonie, de l'omniprésence des "voix», leur modulation, leur imbrication profonde comme trait saillant certes, mais si bien psalmodiée et ne laissant d'essaimer que leur hybridité ne nous apparaît pas au fil de notre première lecture, toujours un peu distraite. C'est là que réside la force incantatoire de l'oeuvre, l'hybridation des voix dont l'imprégnation diffuse n'est justement saisie, ne devient saisissable, "réelle», qu’à la faveur de ce "travail sur la lettre» qu'implique la traduction. Chez Faulkner, cette polyphonie atteint à la virtuosité.

Une leçon que je tire personnellement du chantier de traduction du GRETI est précisément que cette imprégnation subtile, diffuse, polyphonique et contrapunctique, des voix nous est rendue perceptible par les coups de sonde de stratégies de traduction constamment réélaborées à la faveur d'un protocole évolutif redistribuant la donne à force d'abandons et de reconductions appelés à pénétrer la dynamique densément stratifiée des éléments microtextuels auxquels est chevillée la "parlance » de l'œuvre, formant un faisceau qui embrasse aussi bien ses phases de condensation que ses procédés elliptiques.

Une autre conséquence importante de ce travail, à mon sens, est qu'il rend caduque la bipolarisation entre sourciers et ciblistes. Ce projet, me semble-t-il, fut l'occasion de faire l'apprentissage d'une dynamique multipolaire qui interdit de se cantonner dans l'un ou l'autre de ces pôles d'attraction. Comme proposé plus haut, cette série d'opérations ciblées, et d'autres plus fortuites, loin de s'en remettre à un tuteur du genre «troisième code» ou «troisième langue», s'apparente plus volontiers à une triangulation ouverte débordant les polarisations initiales pour former une matrice perméable à divers cas de figure. La raison est simple: nous avons affaire à un matériau très complexe. Je le dirai en bref: avant de considérer l'allégeance ou à la lettre ou au sens dans la traduction, il faut envisager la facture de l'œuvre elle-même. Ainsi, l'excès de littéralité, phénomène décrié, sinon redouté dans l'exercice de la traduction, par crainte qu'il n'étouffe ou écrase le sens, le lamine et entrave sa translation vers un pôle récepteur, y sévit parfois avec une singulière obstination et se retrouve même profondément encodé, de propos délibéré, comme structure fondamentale de l'œuvre, constituant en quelque sorte le seing de sa poétique. C'est le cas notamment avec la «grande prose», au demeurant celle de Faulkner: la littéralité syntaxique est délibérément mise à profit pour brouiller la hiérarchisation des voix dans la trame diégétique, depuis le point de vue de l'instance narratoriale première, extradiégétique - le narrateur omniscient -, en passant par composante dialogale fournie par un narrateur intradiégétique, pour se ramifier enfin dans la polyphonie des sociolectes et diverses isotopies vernaculaires qui émaillent la voix narrative et finissent par l'enchâsser à la façon d'une poupée gigogne dans une structure densément enchevêtrée formant un 
palimpseste, une espèce de grande membrane vibratoire où les voix se répercutent, se font écho et amplifient la force incantatoire de l'œuvre.

En l'occurrence, le respect de la lettre, le souci de littéralité ainsi entendu, n'entame ni ne met en péril les soins apportés pour ménager un point d'impact consonant dans la langue hôte. La charge de sens s'en trouve grandie et amplifiée dans des formes inédites qui octroient une postérité à l'œuvre dont le point de fuite est, tel que l'entendait Walter Benjamin (1923/1997: 14-15), son coefficient de traduisibilité (Übersetzbarkeit). Celui-ci doit être élaboré, car il est "en germe»(keimhaft) dans l'œuvre, et il implique à coup sûr la saisie d'un temps opportun, à l'instar d'un kairos au sens grec, le moment propice où les temps sont mûrs pour une retraduction. Ainsi l'ensemble des stratégies de traduction mises à profit par l'équipe du GRETI reposet-il sur des apprentissages et des solutions tributaires d'un savoir élaboré $a$ posteriori, sur une plate-forme conviant un nombre discret et bien ciblé de «lectures tangentielles» qui, en revanche, n'ont pu être activées et porter fruit que sous le coup d'une sensibilité accrue aux éléments en dormance qui s'éveillent sous la poussée d'une conjoncture propice à leur mise en branle.

Qu'est-ce qu'une conjoncture? L'ensemble des conditions propices à l'élargissement des espaces et des horizons de référence, aussi bien que la dilatation ou la condensation des vecteurs qui convergent et se diffractent au cœur du cône temporel. Par exemple, dans le cas qui nous occupe, la francophonie ne peut désormais plus être confinée à une "gallicité» ancestrale, à moins qu'elle ne soit réinsufflée par les terroirs du Nouveau Monde, ni à la codification empesée des "genres» hexagonaux, car son champ d'action s'est déporté vers des périphéries qui n'en sont plus, les espaces caribéen, maghrébin, québécois, acadien et franco-américain, pour ne nommer que ceux-là. L'hexagone s'est métamorphosé en polygone. Enfin, l'assemblage à géométrie variable tracé par les diverses pistes poursuivies, les stratégies de traduction tantôt abandonnées tantôt redécouvertes par l'équipe du GRETI, ressortissent à un pluralisme méthodologique qui seul permet de cibler les veines porteuses d'une matière vive qui ne peut rester «lettre morte» si l'on entend parvenir à une retraduction décentrée qui sache héberger dans la langue hôte les motivations profondes de la poétique faulknérienne.

Il va sans dire que ce chantier et les diverses avenues qu'il a permis de baliser s'inscrivent dans une constellation de sens plus large, fragmentée et proliférante, qui ne peut désormais plus être jaugée à l'aune des parangons de la «haute culture», apportant un démenti éclatant à une inférence quasi axiomatique formulée par Berman en 1985, selon laquelle, écrivait-il, "malheureusement, le vernaculaire ne peut être traduit dans un autre vernaculaire. Seules les koinai, les langues "cultivées", peuvent s'entretraduire» (Berman 1985/1999: 64). Ce renversement de la donne héritée des polysystèmes littéraires arrimés aux koinai nous découvre une perspective d'une plus grande magnitude et grosse d'horizons insoupçonnés. Au-delà d'une arène traductionnelle cosmopolite où un foisonnement de terroirs et de dialectes se pressent au portail et «creusent la fosse de Babel», pour reprendre ici l'expression de Kafka, il nous faut reconfigurer du tout au tout l'échiquier de nos savoirs et de nos apprentissages et faire brèche vers une épistémologie ouverte. 


\section{Prémisses d'une épistémologie ouverte}

J'aimerais clore cet exposé en décloisonnant l'espace critique où s'inscrit toute recherche pour l'ouvrir à des horizons qui ménagent les avenues d'un pluralisme méthodologique, en l'occurrence les prémisses d'une épistémologie ouverte. C'est une perspective qu'a embrassée d'emblée l'épistémologue d'origine autrichienne Paul Feyerabend (1975/1979, traduit par Jurdant et Schlumberger) dans son retentissant ouvrage, dont le titre à lui seul laisse présager le torrent de controverses et l'atmosphère sulfureuse qui allaient entourer sa sortie: Contre la méthode. Esquisse d'une théorie anarchiste de la connaissance. Cette perspective que d'aucuns ont jugée «cavalière» vient plutôt remettre les pendules à l'heure en ce qui a trait au partage des savoirs et à leur enracinement dans la variété indénombrable des expériences à la faveur desquelles l'espèce humaine apprivoise sa propre condition.

Après avoir étudié avec Popper, avoir défendu dans sa foulée le point de vue de la «contre-induction" opposée à la méthode critique de confirmation des hypothèses du positivisme logique, Feyerabend (1975/1979) va ensuite étendre sa critique à la méthode de corroboration préconisée par Popper à l'appui du critère de réfutabilité, pour ainsi évoluer vers une forme radicalisée de pluralisme méthodologique: une théorie ne peut pas être testée par les seules observations qu'elle engendre et auxquelles elle donne sens; seule une autre théorie, incompatible, ou fortement divergente, peut fournir les éléments susceptibles de la réfuter. De la même façon, Feyerabend va se désolidariser du modèle kuhnien, estimant trop faible chez lui le coefficient des conditions critiques prévalant pour enclencher un changement de paradigme et un renversement du statu quo : la théorie de Kuhn ne peut expliquer la transition d'une science moniste normalisée, d'un corpus unifié de postulats et d'axiomes, à une science pluraliste révolutionnaire, puisque l'impossibilité d'un langage d'observation sémantiquement neutre entre les deux protagonistes fait d'une pluralité de théories alternatives la condition sine qua non pour amorcer cette transition vers une nouvelle ère de la connaissance.

Partisan de l'hédonisme en matière d'épistémologie - pour lui l'expertise du shaman ou du sorcier habitant les forêts de l'Amazonie vaut bien celle du médecin équipé de tout son arsenal technologique et pharmacologique - et fervent défenseur de la prolifération des théories ou d'hypothèses concurrentes, Feyerabend va bientôt reconnaître à ce pluralisme méthodologique une portée ontologique. Dans le chapitre de conclusion de Against Method, il affirme sans ambages: «L'idée que la science peut, et doit, être organisée selon des règles fixes et universelles est à la fois utopique et pernicieuse» (Feyerabend 1975/1979: 332). D’aucuns ont voulu réduire sa pensée au slogan: "Anything goes »: "Tout est bon». Mais il s'agit là d'une piètre caricature du relativisme très ferme et très nuancé qu'il articule de divers points de vue et qui s'enracine dans une résistance farouche à l'endroit du règne de l'évidence, du moins présumée telle, à commencer par le poncif qui veut que la nature détermine le contenu de la pensée. Feyerabend défend plutôt une théorie artéfactuelle, contextuelle et holistique, de la signification. Toute hypothèse réputée valide est un construit social chevillé par une armature axiologique: le contenu de la pensée est un artéfact humain, à l'instar de toute œuvre d'art, tout ustensile ou tout objet symbolisant l'adhésion à une culture ou à une religion. Sa composante parfois densément chiffrée par voie d'analogies filant tout un florilège de métaphores ressortit à un comportement ritualisé qui varie selon les cultures. 
Réactivant à de nouveaux frais la thèse de la relativité linguistique de SapirWhorf, Feyerabend (1975/1979) va pareillement radicaliser la thèse de l'incommensurabilité sémantique des paradigmes énoncée par Thomas Kuhn. Les divers paradigmes balisant le champ du savoir mobilisent des concepts qui ne peuvent pas être réduits aux relations logiques habituelles d'inclusion, d'exclusion ou d'intersection, ils sont aussi nourris par des jugements esthétiques, des préjugés métaphysiques et des options idéologiques et religieuses de divers tenants et diverses extractions. Toute théorie scientifique, aussi objective se prétende-t-elle et aussi abstraite que soit sa forme, s'inscrit dans un horizon historique. En revanche, toute description factuelle repose sur une théorie préalable. Qui plus est, il y a aussi des faits qui ne peuvent être appréhendés sans le secours d'alternatives à la théorie devant être testée, pouvant parfois solliciter un ordre de vision ou un mode de représentation hétérodoxe, voire complètement étranger à l'horizon ontologique dans lequel s'inscrit cette théorie. Pour Feyerabend toute règle méthodologique est associée à des présupposés cosmologiques qui, à leur tour, déterminent ou trahissent l'adhésion à certaines traditions métaphysiques qui elles-mêmes ressortissent à diverses formes de vie liées à des valeurs culturelles et sociales. Les énoncés scientifiques sont des produits humains liés à une sensibilité culturelle et à un environnement cognitif associé à des séquences aléatoires, fortuites et idiosyncrasiques du développement historique.

C'est pourquoi il nous faut consentir à l'existence et à la cohabitation d'une pluralité de points de vue dont l'apport peut emprunter des avenues insoupçonnées et ainsi contribuer à l'élargissement de nos horizons tout en favorisant l'émergence d'une forme plus transparente de rationalité. Justement, s'il est un domaine où l'adoption d'une méthodologie pluraliste peut se révéler d'une grande fécondité, c'est bien celui de la traductologie. L'idée même de reléguer la traduction à une fonction ancillaire la subordonnant à la simple gestion du commerce interlinguistique sur un échiquier formaté par les puissances économiques fait l'impasse sur sa générativité comme vecteur de métamorphoses dans le mouvement global d'acculturation des terroirs. La possibilité de scruter les leviers de son action, de mesurer l'enjeu et la portée de son interaction avec les ressorts de l'expression au gré des constellations culturelles avec lesquelles elle entre en contact et au sein desquelles elle est appelée à graviter oblige à considérer une kyrielle d'aspects et de facteurs qui débordent largement la seule compétence linguistique.

Le momentum qui se concrétise dans la tâche précise à laquelle est assigné tel ou tel autre mandat de traduction est ni plus ni moins que l'épiphanie passagère ou la concentration instantanée de l'image que nous renvoie un microcosme humain désormais décloisonné et ouvert à une migration cosmopolite des savoirs, des modes de vie comme des artéfacts culturels. Son mandat la projette à l'interface des multiples médiations, tractations et interactions qui prolifèrent dans la mouvance de cette économie symbolique dont la gestation s'est enclenchée et a pris son essor il y a plusieurs millénaires en Mésopotamie, lorsque l'on gravait sur des plaquettes d'argile le comput des transactions commerciales ou les rudiments d'une cartographie des corps célestes, ou encore en Chine lorsqu'on interprétait les craquelures sur les carapaces de tortues chauffées à blanc. À cet égard, on ne peut plus s'en tenir à une vision dogmatique de la connaissance qui ferait l'économie d'une sédimentation beaucoup plus riche et diversifiée du champ de l'expérience. Quoique cela puisse paraître difficile à concevoir pour certains, j'estime que les constructions les plus sophistiquées de 
l'esprit humain, par exemple celles qui affichent un haut degré d'abstraction, doivent nécessairement communiquer quelque part avec les mécanismes les plus rudimentaires qui ont présidé à l'éclosion et à l'évolution des diverses formes de vie sur cette planète. De la même façon, maintenir une coupure nette entre les savoirs de pointe gérés par les "communautés d'experts» et le savoir-faire hérité de modes de vie apparemment plus modestes basés sur des rites ancestraux qui sont souvent thésaurisés dans la "mémoire des peuples» me semble une manœuvre inopportune et stérile.

Ma défense et illustration d'un point de vue non déterministe sur la traduction et sur l'exercice du langage en général s'inscrit dans le cadre d'une vision holistique de la connaissance où nul gain cognitif significatif ne peut être enregistré sans en référer à la totalité du champ de l'expérience. Une vue non déterministe en appelle pareillement à une forme ouverte, explicite, de pluralisme méthodologique.

De nature conjecturelle, toute hypothèse n'est valide et viable qu'en considération du fait qu'elle ne saurait épuiser les ressorts de la complexité à laquelle est redevable tout objet d'investigation pouvant nous solliciter dans l'univers au sein duquel nous évoluons. C'est la leçon du faillibilisme que préconise Popper. Cette condition vaut a fortiori pour l'expérience de la traduction et la réflexion qui s'en inspire, précisément parce qu'elles occupent une niche de prédilection, tant pour apprécier la magnitude des différences que procéder à la prospection d'affinités électives assorties à la pluralité irréductible des langues et à la variété indénombrable des contextes culturels, terroirs aussi bien que zones franches d'échanges transfrontaliers et lieux de métissages auxquels elles sont corrélées. À cet égard, l'affirmation de I.A. Richards que j'ai citée en exergue au présent article, pourrait s'avérer beaucoup moins ésotérique qu'il n'y paraît, et la traduction, effectivement, pourrait bien être «le type d'événement le plus complexe à s'être produit dans l'évolution du cosmos».

\section{RÉFÉRENCES}

BAKer, Mona (1998): Réexplorer la langue de la traduction: une approche par corpus. Meta. 43(4):480-485.

Benjamin, Walter (1916/1991): Über Sprache überhaupt und über die Sprache des Menschen. In: Rolf Tiedemann et Hermann ScheppenhëUser, dir. Gesammelte Schriften, Vol. II/1. Frankfurt am Main: Suhrkamp Verlag, 140-157.

Benjamin, Walter (1923/1997): L'abandon du traducteur. Prolégomènes à la traduction des Tableaux parisiens de Charles Baudelaire. (Traduit et annoté par Laurent LAmy et Alexis Nouss) TTR. 10(2):13-62.

Berman, Antoine (1995): Pour une critique des traductions: John Donne. Paris: Gallimard. Berman, Antoine (1985/1999): La traduction et la lettre ou l'auberge du lointain. Paris: Seuil.

BoHr, Niels (1958/1991): Physique atomique et connaissance humaine. (Traduit par Edmond Bauer et Roland Omnès, revue par Catherine Chevalley) Paris: Gallimard.

Brownlie, Siobhan (2003): Berman and Toury: the Translating and Translatability of Research Frameworks. TTR. 16(1):93-120.

Chapdelaine, Annick (2001) Traduire l'incantation de l'œuvre: Le Hamlet de William Faulkner. In: Annick Chapdelaine et Gilian Lane-Mercier, dir. : 83-129.

Chapdelaine, Annick et Lane-Mercier, Gillian, dir. (2001): Faulkner. Une expérience de retraduction. Montréal: Presses de l'Université de Montréal.

Chesterman, Andrew (1997): Memes of Translation: The Spread of Ideas in Translation Theory. Amsterdam/Philadephie: John Benjamins. 
Chesterman, Andrew (2006): Vers une traductologie poppérienne. In: Michel BAllard, dir. Qu'est-ce que la traductologie? Arras: Artois Presses Université, 171-178.

Сномsкy, Noam (1971): Aspects de la théorie syntaxique. (Traduit par Jean-Claude Milner) Paris: Seuil.

Dawkins, Richard (1976): The Selfish Gene. Oxford: Oxford UP.

Eco, Umberto (1962/1965): L'œuvre ouverte [Opera aperta. Forma e indeterminazione nelle poetiche contemporanee] (Traduit par Chantal Roux de Bézieux et André BoucoureCHLIEv) Paris: Seuil.

Eco, Umberto (1990/1992): Les limites de l'interprétation. (Traduit par Myriem Bouzaher) Paris: Grasset.

Feyerabend, Paul (1975/1979): Contre la méthode. Esquisse d'une théorie anarchiste de la connaissance. (Traduit par Baudoin Jurdant et Agnès Schlumberger) Paris: Seuil.

FraWley, William (1984): Prolegomena to a Theory of Translation. In: William FraWley, dir. Translation: Literary, Linguistic, and Philosophical Perspectives. Londres/Toronto: Associated UP, 159-175.

Kant, Immanuel (1781/1975): Critique de la raison pure. (Traduit et annoté par André TremeSAYGUes et Bernard PaCaUd) Paris: PUF.

Kunn, Thomas (1962/1972): La structure des révolutions scientifiques. (Traduit par Laure MeYer) Paris: Flammarion.

LAMY, Laurent (2007): Clavis hermeneutica: la problématisation du traduire chez Schleiermacher ou l'ébauche du paradigme de la compréhension intersubjective. Meta . 52(3):588-602.

Lyotard, Jean-François (1983): Le différend. Paris: Éditions de Minuit.

Nida, Eugene A., et Charles R. TABer (1969): The Theory and Practice of Translation, With Special Reference to Bible Translating. Leiden: Brill.

Popper, Karl R. (1934/1973) : La logique de la découverte scientifique. (Traduit par Nicole ThyssenRutten et Philippe Devaux) Paris: Payot.

Popper, Karl R. (1963/1986): Conjectures et réfutations. La croissance du savoir scientifique. (Traduit par Michelle-Irène De Launay et Marc B. DE Launay) Paris: Payot.

Richards, I. A. (1953): Toward a Theory of Translating. In: Arthur F. Wright, dir. Studies in Chinese Thought. Chicago: University of Chicago Press, 247-262.

Quine, Willard Van Orman (1960): Word and Object. Cambridge: MIT Press.

Toury, Gideon (1995): Descriptive Translation Studies and Beyond. Amsterdam/Philadelphie: John Benjamins.

van Leuven-Zwart, Kitty (1989): Translation and Original: Similarities and Dissimilarities, I. Target. (1)2:151-181.

Venuti, Lawrence (1995): The Translator's Invisibility. A History of Translation. London/New York: Routledge.

Wittgenstein, Ludwig (1960): Schriften: Tractatus Logico-Philosophicus, Tagebücher, Philosophische Untersuchungen. Frankfurt am Main: Suhrkamp Verlag. 